\section{A neuromuscular syndrome caused by abnormalities in reproductive hormones}

This letter is intended to inform general practitioners (GPs), specialist doctors and nurses about a syndrome that causes distress and anxiety, from pain as well as from fear of sinister neurological disease. The syndrome is common when known and looked for. I see an average of two patients a week. The syndrome is, to the best of my knowledge, not yet known.

This neuromuscular syndrome is caused by abnormalities in female reproductive hormones, abnormalities especially precipitated by hormone replacement therapy (HRT) and contraception. The syndrome is distinct from the menopause and, unless recognised, persists for several years. Women aged between 30 and 60 years are thus referred from orthopaedic, plastic and vascular surgeons, from rheumatologists, neurologists and GPs with symptoms of an unknown neuromuscular disease.

From studying 170 patients since 2002 , I can report that the syndrome has the following features:

- Symptoms predominate in one upper limb, affect the non-dominant as often as the dominant, frequently include the same side of the neck and face and the ipsilateral lower limb.

- Symptoms vary between patients but are consistent - even over several years - in an individual and include:

- Painful numbness and sensation of tightness in the upper arm and top of the shoulder, sensation as if an elastic band is present deep inside the arm, restricting grip or extension at the elbow;

- Spasm, often likened to muscles being wrung-up, sensation of crushing over the shins and, by women who have borne children, to contractions of the gravid womb; - Dystonia in the fingers and toes. Fingers lock in flexion and toes override each other. - Twitching, including on one side of the face, and choreiform movements.

- Fatigability.
Despite numbness and fatigability, sensory deficit and actual weakness are minimal. This is an important distinction from the entrapment and generalised neuropathies, radiculopathies, myopathies and degenerative nervous disease, which the syndrome mimics. Many patients have not been diagnosed for several years, many misdiagnosed - frequently as the thoracic outlet syndrome (of fixed anatomical pathology in the neck). Several underwent surgery, unnecessarily.

The syndrome usually develops over 2 weeks, within 4 months of institution, abrupt withdrawal, or change (sometimes to "parallel imports' of the same brand) of the medicinal hormone.

Some $75 \%$ of the women who developed this syndrome were on medicinal reproductive hormones; in $75 \%$ HRT, in $25 \%$ contraceptives including to regulate the menstrual period. Hormones include:

- levonorgestrel-impregnated intrauterine device (IUD)

- estradiol patch

- parenteral progestogen-only contraceptive

- tablets:

- progestogen and oestrogen

- estradiol and norethisterone acetate

- conjugated oestrogens-only estradiol

- standard-strength norethisterone, mestranol

- standard-strength levonorgestrel,

ethinylestradiol.

Twenty-five percent of the women have undergone hysterectomy, in half of whom, total. Eighty percent of those women were also on HRT and the syndrome was often precipitated by changes in HRT. In a few women, sterilisation by ligation of the Fallopian tubes was the principal gynaecological event and, in one, hormones were given to assist change of gender to female.

Electromyography shows abnormal excitability of individual muscle fibres, but no primary muscle disease, no nerve entrapment and no generalised neuropathy. The syndrome is likely due to the effect of hormones on ion channels on muscle and nerve cell membranes.

Diagnosis is made by eliciting association with hormones. As the contraceptive pill, depot njections, HRT and the IUD may not be regarded as medicines, and as onset may have been a few years earlier, the patient's history needs specific questioning including consideration of the patient's diary and/or GP prescription notes. Often the patient herself then suddenly realises the association.

Treatment is by reassurance that the syndrome is not progressive and - if possible - by withdrawal of the hormone. Patients can also be forewarned of the possible neuromuscular symptoms when hormones are instituted. Although empirically hormones themselves can be considered for treatment, as precise assay of all female reproductive hormones is not available and as hormones have the side effects of breast cancer and embolism, hormones are best avoided. Carbamazepine can be tried, as can injection with botulinum toxin into selective underlying muscles where patients point to a distinct zone of pain, spasm or dystonia such as on the side of the neck on in the shoulder.

\section{Elias Ragi, FRCP, DPhil(Oxon)}

Consultant Clinical Neurophysiologist, Royal

Devon and Exeter Hospital, Exeter, UK.

E-mail:eragi@doctors.org.uk

\section{Cytology sampling using brushes}

I wonder if any of the Journal's readers involved with the new cytology sampling using the brushes have experienced unintentional 'removal' of coils?

The new procedure involves rotating the brushes at the cervical os five times clockwise and five times anti-clockwise. Some of my nurses have had near misses.

I advised using a Spencer Wells forceps to keep both coil threads flush against the external cervix to minimise this risk.

Leng Neoh, MRCGP, FFFP

The Thornaby and Barwick Medical Group

Thornaby, Stockton-on-Tees, UK.

E-mail:leng.neoh@nhs.net

NEWS ROUNDUP

\section{Gynaecological cancer information booklet}

WellBeing of Women (WoW) has produced a free information booklet on gynaecological cancers as part of their 'Low Down on Down There' gynaecological cancer awareness campaign. The drive is to encourage women to be aware of the signs and symptoms of the most common gynaecological cancers. Written by Professor Sean Kehoe, FRCOG, Professor of Gynaecological Cancer at John Radcliffe Hospital in Oxford, UK the booklet covers ovarian, cervical, endometrial and vulval cancer. The key messages of the campaign are:

- Know your norm - be aware of any changes in your body (i.e. bleeding between periods, bloating and abnormal discharge)

- Regularly attend routine screening

- Be aware of the symptoms of gynaecological cancers

- Seek medical attention as soon as possible if unsatisfied with the diagnosis, do not be afraid to ask for a second opinion

- There's no need to be afraid - the earlier a cancer is found, the easier it is to treat.

Copies of the booklet can be requested by telephoning the Supporter Services Team on +44 (0)20 77726400 or alternatively by e-mailing wellbeingofwomen@rcog.org.uk.

\section{Adult male circumcision and HIV risk}

The National Institute of Allergy and Infectious Diseases (NIAID), part of the National Institutes of Health $(\mathrm{NIH})$, announced an early end to two clinical trials of adult male circumcision because an interim review of trial data revealed that medically performed circumcision significantly reduces a man's risk of acquiring HIV through heterosexual intercourse. The trial in Kisumu, Kenya of 2784 HIV-negative men showed a $53 \%$ reduction of HIV acquisition in circumcised men relative to uncircumcised men, while a trial of 4996 HIVnegative men in Rakai, Uganda showed that HIV acquisition was reduced by $48 \%$ in circumcised men. Both trials involved adult, HIV-negative heterosexual male volunteers assigned at random to either intervention (circumcision performed by trained medical professionals in a clinic setting) or no intervention (no circumcision). All participants were extensively counselled in HIV prevention and risk reduction techniques

For more information on the Kenyan and Ugandan trials of adult male circumcision, see the NIAID Questions and Answers document at http://www3.niaid.nih.gov/news/QA/AMC12_Q A.htm.

\section{Doctors warn of cervical screening decline}

Stark falls in the number of women coming forward for cervical screening could lead to an increased rate of cervical cancer say doctors. Only $69.4 \%$ of women aged between 25 and 29 years who were invited for screening attended in $2005 / 2006$ compared to the figure of almost $80 \%$ of women in 2005. The NHS Cancer Screening Programme said that a similar trend had been observed among women aged between 30 and 34 years. In some ways the screening programme has become a victim of its own success, with the public now having a low level of awareness about cervical cancer. Since a national screening programme was launched in 1988, the number of deaths attributed to the disease has fallen from 6000 to 1000 every year. Director of the NHS Cancer Screening Programme, Julietta Patnick, said that initial research suggested that some women didn't attend screening because they were concerned that it may be embarrassing or painful.

1803939

Reported by Henrietta Hughes, MRCGP, DFFP General Practitioner, London, UK 